\title{
Einfluß der Vitamine $A, E$ und $C$ auf die Wirkung von Überträgersubstanzen des vegetativen Nervensystems bei Kaninchen mit künstlicher Leberschädigung
}

\author{
Von G. O. SCHLÜTZ1ㄱ) und J. VeNULET²) \\ Aus dem Center of Research, University of Damascus, Syria (Director: Professor Dr. med. G. O. Scblütz)
}

(Eingegangen am 10. Oktober 1964)

\begin{abstract}
Ausgehend von der Beobachtung, daß bei Kaninchen mit $\mathrm{CCl}_{4}$-induziertem Leberschaden auf der Höhe der Parenchymschädigung (Transaminasen 300-1000\% erhöht) und beim Ausbleiben der Pyrogenwirkung des Lipopolysaccharids von E. Coli die Ansprechbarkeit von Adrenalin und Noradrenalin erhöht ist - wobei zu gleicher Zeit eine gering herabgesetzte Ansprechbarkeit auf Acetylcholin beobachtet wird - wurde der Einfluß der Vitamine $\mathrm{A}, \mathrm{E}$ und $\mathrm{C}$ auf die Blutdruckwirkung der genannten Überträgersubstanzen an $\mathrm{CCl}_{4^{-}}$geschädigten Kaninchen $(1 \mathrm{ml} / \mathrm{kg})$ untersucht. Dabei wurde Vitamin A gewählt, weil ihm im allgemeinen eine Leberschutzwirkung zugeschrieben wird. Vitamin E wurde aufgrund seiner Beteiligung am Elektronentransport gewählt, und Vitamin $C$ wegen eines in den Zellen von Leber, Niere und Nebennieren vorkommenden mikrosomalen ascorbinsäureabhängigen Flavin-Cytochrom- $b_{5}$-haltigen Lipoprotein-Elektronentransportsystems, das mit Sicherheit an der enzymatischen $\beta$-Hydroxylierung von Dopamin zu Noradrenalin beteiligt ist. Die Ergebnisse werden einzeln abgehandelt und diskutiert.
\end{abstract}

In rabbits with $\mathrm{CCl}_{4}$-induced liver parenchyma damage (transaminases increased $300-1000 \%$ ), in which $E$. Coli lipopolysaccharides do not exert pyrogenic activity, the response to adrenaline and noradrenaline is increased, while the response to acetylcholine is slightly decreased. Starting from this observation, the influence of vitamins $\mathrm{A}, \mathrm{E}$ and $\mathrm{C}$ on the action of the above hypertensive humoral agents was studied in $\mathrm{CCl}_{4}$-damaged rabbits $(1 \mathrm{ml}$. $/ \mathrm{kg}$ ). Vitamin $A$ was chosen, because a liver-protective function is generally ascribed to it. Vitamin $E$ was chosen, because it is involved in electron transport, and vitamin $C$ because a lipoprotein electron transport system, containing an ascorbic acid-dependent, flavin-cytochrom- $b_{5}$-system, occurs in the microsomes of the cells of liver, kidney and adrenal gland, and is certainly concerned in the enzymic $\beta$-hydroxylation of dopamine to noradrenaline. The individual results are described and discussed.

Ausgehend von der Beobachtung, daß bei mit $\mathrm{CCl}_{4}$ induziertem Leberschaden auf der Höhe der Parenchymschädigung und kurze Zeit nach dem Gipfelpunkt der überhöhten Transaminasenwerte von über $300 \%$ die Zufuhr des pyrogenen Faktors von E.Coli ohne Fieberreaktion erfolgt, sowie basierend auf der Tatsache, daß die Cholinesterasebestimmung als Leberfunktionstest angewandt wird, kam uns der Gedanke, den Reaktionsausschlag der Blutdruckwirkung von Adrenalin, Noradrenalin und Acetylcholin, die intravenös verabreicht wurden, näher $z u$ untersuchen (1). $\mathrm{Da}$ die diese Substanzen abbauenden Enzyme sich vorwiegend in der Leber befinden, liegt der Gedanke nahe, $\mathrm{da} B$ bei Leberschädigung eine andere Wirkungsstärke dieser Überträgetstoffe des vegetativen Nervensystems beobachtet werden kann.

Da dem Vitamin A im allgemeinen eine Leberschutzwirkung zugesprochen wird, wie besonders aus den Arbeiten von MeunIer und Mitarbeitern (2) mit Benzoatintoxikationsversuchen hervorgeht, und fußend auf den Beobachtungen von Gallagher und Simmonds (3), daß Nicotinsäure oder d,1-Tryptophan drei Tage vor $\operatorname{der} \mathrm{CCl}_{4}$-Vergiftung täglich zugeführt vor dessen Giftwirkung erheblich schützt, sowie der weiteren Feststellung Gallaghers (4), daß Vitamin E in Dosen von $95 \mathrm{I}$. U. intraperitoneal zugeführt, 15 Ratten, die

1) Nach einem Vortrag des Verfassers auf der „Second National Conference of the Bulgarian Society For Physiological Sciences" Sofia, 28. 9.-3. 10. 1964.

2) Department of Pharmacology; ständige Anschrift: Drug Research Institute, Warschau 36. mit $4 \mathrm{~m} l \mathrm{CCl}_{4}$ oral vergiftet wurden, vollkommen gegen den Tetrachlorkohlenstoff schützte, ließ uns Versuchsreihen ansetzen mit Kaninchen, die vor der $\mathrm{CCl}_{4}$-Schädigung die Vitamine $\mathrm{A}, \mathrm{E}$ und $\mathrm{C}$ erhielten. Für das Vitamin $\mathrm{E}$ interessierten wir uns besonders, weil eine große Ähnlichkeit im Erscheinungsbild der Leberschädigung sowohl bei der Tetrachlorkohlenstoffvergiftung wie auch beim Vitamin E-Mangel beobachtet wird (5): Der Schaden beginnt hauptsächlich an der Membranseite; des weiteren auch wegen seiner Beteiligung am Elektronentransport. Das Vitamin $C$ erregte unser besonderes Interesse wegen eines in den Zellen von Leber, Niere und Nebennieren vorkommenden mikrosomalen, ascorbinsäureabhängigen Flavin-Cytochrom- $b_{5}$-haltigen Lipoprotein-Elektronen-Transportsystems, das mit Sicherheit mit der enzymatischen $\beta$ Hydroxilierung von Dopamin zu Noradrenalin in Verbindung steht (6).

\section{Versuche}

\section{Tiermaterial und Untersuchungsverfabren}

Als Versucbstiere wurden Kaninchen gewählt, die in 6-er-Gruppen eingeteilt wurden:

(1) Vollkontrolle

(2) $\mathrm{CCl}_{4}$-Kontrolle $(1 \mathrm{~m} / \mathrm{kg})$ subcutan

(3) Vitamin A $(30000 \mathrm{I}$. U. $/ \mathrm{kg})+1 \mathrm{ml} \mathrm{CCl}_{4}$ subcutan

(4) Vitamin E $(50 \mathrm{mg} / \mathrm{kg})+1 \mathrm{ml} \mathrm{CCl} / \mathrm{kg}$ subcutan

(5) Vitamin C $(200 \mathrm{mg} / \mathrm{kg})+1 \mathrm{ml} \mathrm{CCl} / 4 / \mathrm{kg}$ subcutan

Die Vitamine wurden 48 Stunden vor der $\mathrm{CCl}_{4}$-Vergiftung verabfolgt: 
Vitamin A: ölige Lösung intramusculär

Vitamin E: wässerige Suspension subcutan

Vitamin C: wässerige Lösung subcutan in Form von Natriumascorbinat.

Adrenalin, Noradrenalin und Acetylcholin wurden intravenös verabfolgt und die Ausschläge kymographisch in Urethananaesthesie registriert. Die einzelnen Gaben waren folgende:

Adrenalin: $A_{1}=1,5 \mu \mathrm{g} / \mathrm{kg} ; \mathrm{A}_{2}=7,5 \mu \mathrm{g} / \mathrm{kg}$.

Noradrenalin: $\mathrm{N}_{1}=2,5 \mu \mathrm{g} / \mathrm{kg} ; \mathrm{N}_{2}=12,5 \mu \mathrm{g} / \mathrm{kg}$.

Acetylcholin: $\mathrm{C}_{1}=0,12 \mu \mathrm{g} / \mathrm{kg} ; \mathrm{C}_{2}=0,60 \mu \mathrm{g} / \mathrm{kg}$.

Die Reibenfolge der Zufuhren waren $\mathrm{A}_{1}-\mathrm{C}_{2}-\mathrm{N}_{1}$ $\mathrm{A}_{2}-\mathrm{C}_{1}-\mathrm{N}_{2}-$. - Biochemisch wurden die Transaminasenaktivitäten von SGPT und SGOT vor und nach der $\mathrm{CCl}_{4}$-Schädigung bestimmt. Tiere mit erhöhten Transaminasewerten wurden ausgeschieden.

\section{Ergebnisse}

Die Ergebnisse sind aus den graphischen Darstellungen leicht ersichtlich. Im Generellen können sie folgendermaßen zusammengefaßt werden:

\section{A. Sterblichkeitsrate}

Die Sterblichkeitsrate der Kaninchen mit Tetrachlorkohlenstoffvergiftung liegt bei $25 \%$. - Die Sterblichkeitsrate der Kaninchen mit $\mathrm{CCl}_{4}$-Vergiftung nach vorheriger intramusculärer Vitamin A-Zufuhr liegt bei $33 \%$. - Die Sterblichkeitsrate der Kaninchen mit $\mathrm{CCl}_{4}$-Vergiftung nach vorheriger subcutaner Zufuhr von wässeriger Vitamin E-Suspension liegt bei $0 \%$. Die Sterblichkeitsrate der Kaninchen mit $\mathrm{CCl}_{4}$-Vergiftung nach vorhergehender subcutaner Zufuhr von wässeriger Natriumascorbinatlösung liegt bei $66,6 \%$.

\section{B. Die Tetracblorkoblenstoffscbädigung}

charakterisiert sich 1. durch erhöhte Mortalität; 2. durch hohe Transaminasenwerte; 3 . durch erhöhte Ansprechbarkeit auf Adrenalin und Noradrenalin sowie gering herabgesetzte Ansprechbarkeit auf Acetylcholin.

\section{Nach Vitamin $A-Z$ ufubr}

Die Tetrachlorkohlenstoffschädigung nach 48-stündiger vorhergehender intramusculärer Zufuhr von $30000 \mathrm{I}$. U. Vitamin $\mathrm{A} / \mathrm{kg}$ ist charakterisiert 1. durch mäßig erhöhte

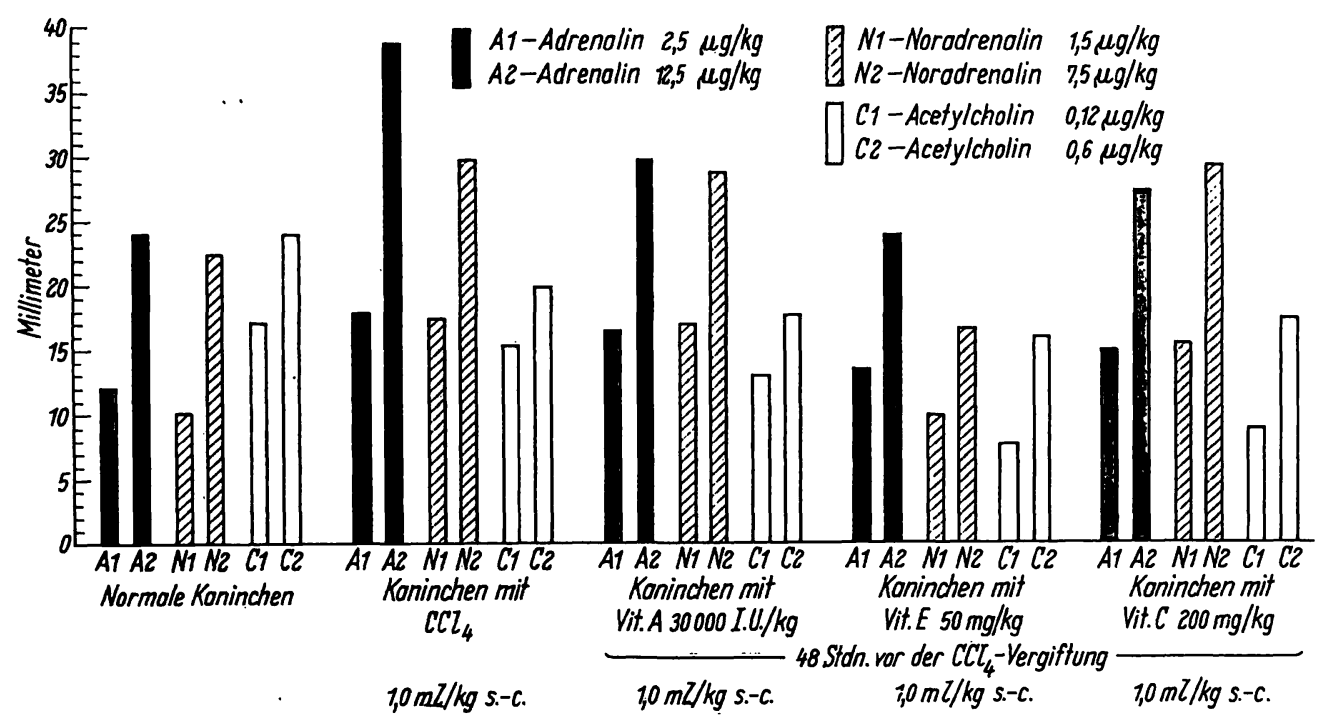

Abb. 1

Höhe der Ausschläge von Uberträgersubstanzen des vegetativen Nervensystems bei Kaninchen

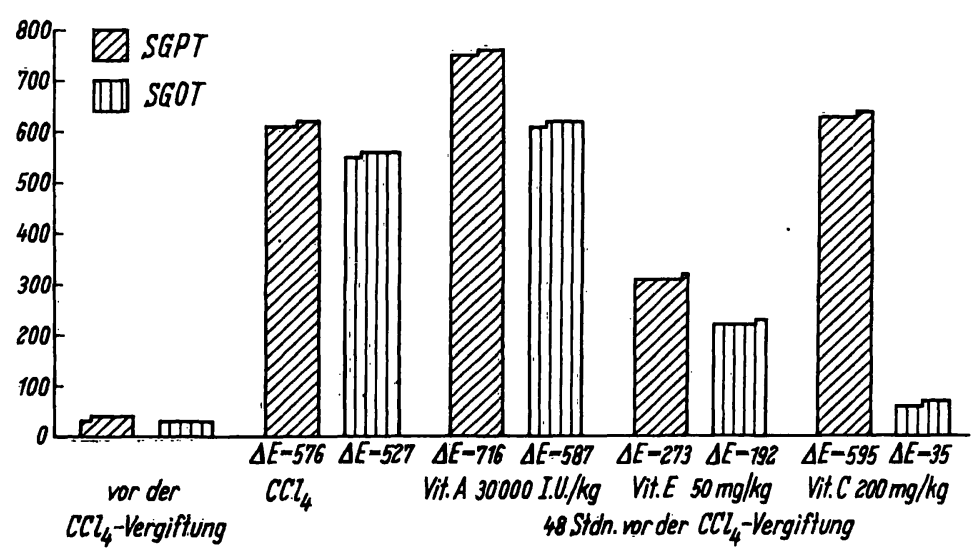

Abb. 2

Transaminasewerte der verschiedenen Kaninchenversuchsgruppen vor und $72 \mathrm{Stdn}$. nach der $\mathrm{CCl}_{4}$-Zufuhr $1,0 \mathrm{~m} / / \mathrm{kg}$. s.-c. 
Mortalität gegenüber der $\mathrm{CCl}_{4}$-Gruppe; 2. durch leicht erhöhte Transaminasenwerte im Vergleich zur $\mathrm{CCl}_{4}$ Kontrolle; 3. durch erhöhte Ansprechbarkeit auf Adrenalin und Noradrenalin (gleiche Höhe wie bei der $\mathrm{CCl}_{4}$-Gruppe), wobei jedoch die Ansprechbarkeit auf Acetylcholin sichtbar verringert ist.

\section{Nacb Vitamin E-Zufubr}

Die Tetrachlorkohlenstoffschädigung nach 48-stündiger vorhergehender subcutaner Zufuhr von $50 \mathrm{mg}$ wässeriger Suspension Vitamin E/kg manifestiert sich 1. durch eine eindeutige Hemmung der $\mathrm{CCl}_{4}$-Mortalitätsrate: $0 \%$; 2. durch mäßig erhöhte Transaminasenwerte (etwa $50 \%$ der $\mathrm{CCl}_{4}$-Werte); 3. durch Erhaltung der Ansprechbarkeit auf Adrenalin und Noradrenalin auf der gleichen Höhe der Vollkontrollen, jedoch ist die Ansprechbarkeit gegenüber Acetylcholin im Vergleich zu den Vollkontrollen bedeutend herabgesetzt. (Die Ausschläge sind noch kleiner als bei der reinen $\mathrm{CCl}_{4}$-Gruppe.) Ebenso wird bei alleiniger Zufuhr von Vitamin E eine geringere Höhe der Ausschläge beobachtet (Vitamin E $+\mathrm{CCl}_{4}$ bedeutend geringer).

\section{E. Nacb Vitamin C-Zufubr}

Die Tetrachlorkohlenstoffschädigung nach vorhergehender 48-stündiger subcutaner Zufuhr von $200 \mathrm{mg}$ Vita$\mathrm{min} C / \mathrm{kg}$ in Form von Natriumascorbinat charakterisiert sich 1. durch erhöhte Mortalität: $662 / 3 \% ; 2$. durch hohe SGPT-Werte wie bei der $\mathrm{CCl}_{4}$-Gruppe, wobei jedoch die SGOT-Werte nur geringfügig erhöht sind; 3. durch erhöhte Ansprechbarkeit auf Adrenalin und Noradrenalin bei herabgesetzter Ansprechbarkeit auf Acetylcholin.

\section{Diskussion}

Ohne weiter auf die zur Zeit bestehenden Theorien der $\mathrm{CCl}_{4}$-Schädigung der Leber einzugehen, können wir ein vollkommen verschiedenes Verhalten des Tiermaterials beobachten. So stellten wir erneut - wie schon in früheren Versuchen (1) - fest, daß die Damaszener Kaninchen im Gegensatz zu den europäischen auf dieselbe $\mathrm{CCl}_{4}$-Gabe $(1,5 \mathrm{~m} / \mathrm{kg})$ mit bedeutend erböbter Enzymfreisetzung antworteten: Europäische Kaninchen: etwa $300 \%$ erhöhte Transaminasenwerte; Damaszener Kaninchen: etwa $1000 \%$ erhöhte Transaminasenwerte. Der Grund hierfür kann in der unterschiedlichen Ernährung gesucht werden: Europäische Kaninchen:
Englische Preßfutterdiät Nr. 47; Damaszener Kaninchen: Vorwiegend Grasfütterung.

Des weiteren war bei den hiesigen Kaninchen bei der $\mathrm{CCl}_{4}$-Dosis von $1,5 \mathrm{~m} / / \mathrm{kg}$ die Mortalitätsrate so hoch, $\mathrm{da} \beta$ wir uns gezwungen sahen, auf Gaben von $1 \mathrm{~m} / / \mathrm{kg}$ herabzugehen. Auch bei dieser Versuchsmenge lag die Sterblichkeitsrate mit $25 \%$ um etwa $300 \%$ höher als bei den europäischen Tieren. Ebenso waren dieselben auch den Überträgersubstanzen gegenüber erheblich empfindlicher, so $\mathrm{da} \beta$ die hier angegebenen Werte die halbe Dosis der anfangs verabfolgten Mengen darstellen. Man kann aus diesen Ergëbnissen ersehen, wie grundverschieden die Laboratoriumstierwelt in verschiedenen Kontinenten und vor allem unter den verschiedensten örtlichen Bedingungen sich verhält besonders, wenn es sich nicht um Tiere aus eigener Zucht, sondern - wie in unserem Falle - um Tiere handelt, die auf dem Wochenmarkt erstanden wurden.

Die Ergebnisse der Vitamin E-Versuche entsprachen unseren Erwartungen. Jedoch überraschten uns die Resultate der Vitamin A-Experimente erheblich. Möglicherweise erstreckt sich dessen Schutzwirkung auf die Aktivierung wesentlicher Enzymsysteme im Prozeß der Glucuronsäuresynthese und der Konjugation wie der Uridyl- oder der Glucuronyltransferase, oder auch auf die Aktivierung der Glycyltransferase, eine Forschungsrichtung, die erregend und vielversprechend erscheint. Die Ergebnisse der Vitamin C-Versuche sind verwirrend, vor allem hinsichtlich der so stark erhöhten Mortalitätsrate, für die wir im Augenblick keine begründete Erklärung geben können. Erhöhte Wirkungsstärke des Noradrenalins hatten wir erwartet und auch in Experimenten allein mit Vitamin $\mathrm{C}$ ohne $\mathrm{CCl}_{4}$-Vergiftung gefunden. $\mathrm{Ob}$ die erhöhte Adrenalinwirkung aufgrund einer Beeinflussung der Monoaminooxydase zustande kommt, muß in weiteren Versuchen geklärt werden, desgleichen das Verhalten gegenüber Acetylchòlin. - Abschließend können wir nur sagen, daß noch eine Vielzahl von Experimenten notwendig ist, um absolut bindende Schlüsse aus diesen Beobachtungen ziehen zu können. Wir werden in Kürze über weitere Untersuchungen in dieser Richtung an dieser Stelle berichten.

Die Verfasser möchten nicht versäumen, an dieser Stelle der Fa. Boehringer \& Söhne, Mannheim-Waldhof, für die Úberlassung von Testkombinationen zu Enzymaktivitätsbestimmungen ihren besonderen Dank auszusprechen.

\section{Literatur}

1. Schlütz, G. O. und J. Venulet, First Meeting of the Federation of European Biochemical Societies, London. March 23, 1964. - 2. Meunier, P., R. Ferrando, J. Jouanneteau und G. Thomas, C. R. hebd. Acad. Séances Sci. 228, 1254 (1949). - 3. Gallagher, C. H. und R. A. Simmoinds, Nature (London)
184, 1407 (1959). - 4. Gallagher, C. H., Nature (London) 192, 881 (1961). - 5. Tappex, A. L. und M. V. Dianzani, Ciba Foundation Symposium, Lysosomes, Churchill LTD., S. 354f. (1963). - 6. Staudinger, HJ., Symposion der DGE über Ascorbinsäure, 2./3. 4. 1964 Mainz, im Druck.

Professor Dr. med. G. O. Schlütz

Center of Research, University of Damascus Damascus, Syria

Ständige Anschrift:

Med.-Chem. Laboratorium Dr. Schlütz

78 Freiburg i. Br. 